\title{
Las palmas de México: presente y futuro
}

\author{
HERMILO J. QUERO \\ Jardín Botánico, Instituto de Biología, UNAM. Apdo. Postal 70-614, Coyoacán. 04510 México, D.F.
}

\begin{abstract}
Resumen. Se menciona la distribución de las palmas en México, sus patrones de asociación con otras plantas y los diversos tipos de vegetación donde están presentes. Se destaca el hecho de que algunas especies han disminuido sus poblaciones, mientras que otras las han visto incrementadas. Las palmas tienen una gran diversidad de usos, principalmente entre la población rural, pero no existe una planeación en el manejo de estos recursos. Se hacen algunas recomendaciones para favorecer algunas poblaciones silvestres, como la de iniciar cultivos comerciales para evitar la sobreexplotación de algunas especies. Se sugiere iniciar estudios sobre algunas palmas de uso potencial o subutilizadas.

Abstract. The distribution and the habitat of Mexican palms are described. It is pointed out that the populations of some species have declined while others have increased. Palms have been empirically used by local people for a variety of purposes and sometimes this has led to the depletion of the natural populations. Several proposals for the recovering and the sustainable management of wild populations are made. The importance of the agrosilvicultural management of palms is stressed. It is necessary to conduct detailed research on underutilized palm species that have a promising economic value.
\end{abstract}

\section{INTRODUCCIÓN}

En México, como en la mayor parte del mundo, el hombre utiliza a las palmas de muy diversas maneras; en algunos casos, las usa directamente ya sea como alimento, construcción o abrigo, o bien para la elaboración de algún producto que le sirva como fuente de ingresos; algunas otras palmas tienen uso ornamental en casas, parques y jardines. Podemos mencionar que en México, de una u otra manera, prácticamente todas las palmas son utilizadas (Cuadro 1).

Las palmas en México tienen una distribución variada, tanto altitudinal como latitudinalmente. En el primer caso, la mayoría de las palmas se encuentran al nivel del mar o a bajas altitudes. Sin embargo, algunas pocas se encuentran en altitudes medias como Brahea nitida que alcanza más de 2 mil metros sobre el nivel del mar en vegetación de bosque de pino-encino y Sabal mexicana que alcanza altitudes cercanas a los 2 mil metros sobre el nivel del mar en bosque de encinos. Por lo general, se considera que las palmas son tropicales latitudinalmente. Sin embargo, en México encontramos algunas especies que alcanzan los $32^{\circ} \mathrm{N}$ por el lado del Pacífico, como es el caso de Washingtonia filifera y Erythea spp., mientras que en la vertiente del Golfo, Brahea berlandieri y Sabal mexicana llegan a latitudes de $28^{\circ} \mathrm{N}$.

En cuanto a la asociación de las palmas con otras plantas, en algunos casos las encontramos formando parte importante de ciertos tipos de vegetación, y en otros pueden formar palmares casi puros, tanto de naturaleza primaria como favorecidos por la acción del hombre. En cualquier caso, estas asociaciones no se presentan de una manera continua, sino que generalmente forman agrupaciones de tamaño variable.

Un ejemplo interesante de asociaciones de palmas, es el que se presenta en la Península de Yucatán, en donde
Coccothrinax readii («knacás») y/o Thrinax radiata («chit») son abundantes en el estrato medio de selvas medianas o bajas subcaducifolias de la vertiente del Caribe, en el estado de Quintana Roo. Otro ejemplo que se encuentra en la misma Península es el de Cryosophila argentea («guano kum») que es el dominante del estrato medio de algunas selvas medianas subperennifolias de Campeche y Quintana Roo.

El género Gaussia también es un ejemplo de palmas importantes en la fisonomía de algunas selvas. G. maya es un componente conspicuo de la selva mediana perennifolia del sur de Quintana Roo, mientras que G. gomez-pompae destaca en las selvas medianas subperennifolias de los alrededores de Tuxtepec, Oaxaca. Otra palma importante en la fisonomía de selvas es Astrocaryum mexicanum, que ocupa el estrato inferior de selvas altas y medianas perennifolias o subperennifolias, tanto en suelos de origen ígneo como de origen calizo en diversas regiones de Veracruz, Tabasco, Oaxaca y Chiapas.

Un caso peculiar es el del género Sabal, cuyas especies se presentan generalmente acaules y con hojas enormes cuando están en alguna selva poco perturbada. Pero cuando dicha selva es alterada, principalmente para hacer potreros mediante incendios periódicos, estas palmas alcanzan alturas considerables y llegan a constituir palmares bastante densos. Tal es el caso de Sabal yapa en gran parte de la Península de Yucatán, Sabal mauritiiformis en Quintana Roo, Campeche, Tabasco y sur de Veracruz y Sabal mexicana, que es la especie más abundante del género en México, y que se encuentra principalmente hacia la vertiente del Golfo, a bajas altitudes, aunque la he encontrado, si bien no tan abundante, en altitudes cercanas a $1052000 \mathrm{~m}$ en el estado de San Luis Potosí. También forma palmares importantes en Oaxaca y Chiapas. 
Cuadro 1. Géneros de palmas silvestres de México.

\begin{tabular}{|c|c|c|c|}
\hline Género & $\begin{array}{l}\text { Número de } \\
\text { especies en } \\
\text { el mundo }\end{array}$ & $\begin{array}{l}\text { Número de } \\
\text { especies en } \\
\text { México }\end{array}$ & Usos \\
\hline Acoelorraphe & 1 & 1 & Techado y construcción \\
\hline Acrocomia & 20 & 1 & $\begin{array}{l}\text { Techado, construcción, comestible, } \\
\text { bebida, medicina popular. }\end{array}$ \\
\hline Astrocaryum & 47 & 1 & Construcción, comestible. \\
\hline Bactris & 100 & 2 & Jarciería, muebles, comestible. \\
\hline Brahea & 5 & 5 & Techado, artesanías, comestible. \\
\hline Calyptrogyne & 5 & 1 & Ornamental \\
\hline Chamaedorea & 100 & 48 & Ornamental, comestible, medicina popular \\
\hline Coccothrinax & 49 & 1 & Techado, construcción, escobas, ornamental. \\
\hline Cryosophila & 7 & 2 & Techado, escobas. \\
\hline Desmoncus & 20 & 2 & Jarciería, muebles, comestible. \\
\hline Erythea & 7 & 7 & Techado, comestible, ornamental. \\
\hline Gaussia & 5 & 2 & No se conoce uso. \\
\hline Geonoma & 70 & 2 & Techado, comestible, ornamental \\
\hline Orbignya & 20 & 2 & $\begin{array}{l}\text { Techado, comestible, aceites, jabones, } \\
\text { artesanías, ornamental. }\end{array}$ \\
\hline Pseudophoenix & 4 & 1 & Ornamental. \\
\hline Reinhardtia & 6 & 2 & Ornamental. \\
\hline Roystonea & 12 & 2 & Forraje, ornamental. \\
\hline Sabal & 14 & 7 & Techado, construcción, comestible, artesanías, forraje. \\
\hline Scheelea & 28 & 2 & Techado, construcción, comestible, aceites. \\
\hline Synechanthus & 2 & 1 & No se conoce uso. \\
\hline Thrinax & 7 & 1 & $\begin{array}{l}\text { Techado, construcciones diversas, } \\
\text { escobas, ornamental. }\end{array}$ \\
\hline Washingtonia & 2 & 2 & Ornamental. \\
\hline
\end{tabular}

Entre otros palmares que se ven favorecidos por el disturbio humano, merecen ser mencionados los formados por algunas especies de Brahea, Orbignya y Scheelea.

Brahea dulcis es la especie más abundante y tiene una distribución muy amplia. Como todas las especies de este género, se le encuentra en suelos calizos en altitudes superiores a los 800 metros, y forma asociaciones casi puras en terrenos perturbados. Otras especies del género que llegan a formar asociaciones importantes son $B$. nitida en Guerrero, Oaxaca y Chiapas, casi siempre asociada con vegetación de bosque de pino-encino, y $B$. decumbens en San Luis Potosí asociada con bosque de encinos.

El género Orbignya está representado en México por sólo dos especies, ambas favorecidas por el hombre. $O$. cohune llega a formar manchones casi puros cuando la vegetación primaria ha sido eliminada y crece en suelos profundos y bien drenados de la porción sur de Quintana Roo. Lo mismo ocurre con $O$. guacuyule en la vertiente del Pacífico, desde Oaxaca hasta Nayarit, aunque también crece de una manera discontinua siendo más abundante en Colima, Jalisco y Nayarit. Desafortunadamente los palmares naturales de esta palma, están siendo sustituidos por palmares de cocoteros.

Un género parecido fisonómicamente a Orbignya es el Scheelea cuyas especies, al igual que las del primero, llegan a formar palmares muy extensos. Esto sucede principalmente con S. liebmannii, que es muy abundante en las planicies de la vertiente del Golfo, desde el sur de Tamaulipas hasta el sur de Campeche, en suelos arcillosos que en algunos casos pueden llegar a inundarse. Estos palmares son de naturaleza secundaria, favorecidos por el hombre aunque en algunos casos forma parte de selvas altas perennifolias. Otra especie del género es $S$. preussii, que forma palmares menos densos que S. liebmannii y sólo se encuentra en el estado de Chiapas.

Acrocomia mexicana es la única especie del género en México. Es una de las palmas que se ve más favorecida por el disturbio humano, por ello es una de las especies de mayor distribución y se presenta en muy diversas condiciones ambientales. Sin embargo, no es común que llegue a formar grandes palmares.

El género Desmoncus tiene pocas especies en México. Se caracterizan por ser trepadoras muy espinosas, y son húmedas del sureste del país.

Entre los palmares de naturaleza primaria en México, destacan los que se presentan en la Península de Yucatán, en las regiones de dunas. En la costa norte del estado de Yucatán se presenta un palmar constituido por Pseudophoenix sargentii, Thrinax radiata y Coccothrinax readii, aunque en ciertas áreas las dos últimas especies son las constituyen- 
tes de ese palmar, como ocurre en el noroeste de Yucatán y en las costas de Quintana Roo. Es interesante mencionar también que Thrinax radiata forma un palmar casi puro en la costa oriental de la Isla de Cozumel.

Acoelorraphe wrighthii constituye otra asociación primaria muy abundante en terrenos inundables y en sabanas de la Península de Yucatán, Tabasco, Chiapas y sur de Veracruz. Cuando esta palma se presenta en sabanas, sus individuos se encuentran formando grupos numerosos semejando islotes, cuya abundancia se ve favorecida por los incendios frecuentes en estas áreas.

Otros palmares primarios presentes en México son los formados por Washingtonia filifera en Baja California y W. robusta en Sonora, Baja California Sur y Baja California. Estos palmares se presentan en manchones aislados, creciendo en lugares donde existen algunos brotes de agua o en cañadas.

Existen palmas que se encuentran en otros tipos de vegetación pero que no forman parte importante de la fisonomía de los mismos, como las pertenecientes a los géneros Chamaedorea, Bactris, Reinhardtia, Geonoma y Synechanthus. Las especies de estos géneros son pequeñas y ocupan los estratos inferiores de selvas, generalmente medianas o altas y de bosques mesófilos.

\section{ESTADO ACTUAL DE LAS PALMAS EN MÉXICO}

Las poblaciones naturales de algunas palmas se han visto disminuidas, mientras que las de otras han aumentado. Sin embargo, la disminución es la situación que se presenta con mayor intensidad. La desaparición o disminución de las poblaciones de palmas se ha debido a factores externos, más que a problemas intrínsecos de las mismas, principalmente por la actividad del hombre, ya sea por la sobreexplotación directa de las poblaciones o por la aiteración o destrucción indirecta de la vegetación natural donde crecen estas palmas. Esta acción del hombre ha provocado que actualmente algunas especies se encuentren amenazadas o en peligro de extinción.

Entre los géneros cuyas poblaciones se han incrementado están aquellos que forman los palmares secundarios de Brahea, Scheelea, Orbignya, Sabal, Acrocomia, etc., ya mencionados. Como ejemplos de palmas cuyas poblaciones se han visto disminuidas por la explotación directa, está el caso de Pseudophoenix sargentii, que crece en la costa norte de Yucatán y en la costa de Quintana Roo. Esta especie tiene uso ornamental. Hace unos 12 años sólo se usaba en parques y jardines de poblaciones cercanas a su medio natural. Actualmente esta práctica se ha extendido a todas las principales urbes de la península, llegando inclusive a algunas ciudades del estado de Tabasco. Dado que los horticultores no están propagando esta palma porque tarda mucho en tener un tamaño adecuado para su venta, les resulta más fácil recolectarla provocando una disminución de las poblaciones naturales, por tanto ésta es una especie vulnerable o amenazada de extinción.
Ejemplos de palmas cuyas poblaciones se han visto disminuidas, tanto por explotación directa como por la destrucción de sus hábitats naturales son Coccothrinax readii y Thrinax radiata, ambas en la Península de Yucatán. Los troncos de Coccothrinax readii son usados para hacer cercas, para construir paredes rústicas tanto de casas rurales como de cabañas, o para edificaciones en centros turísticos y como adorno en algunos hoteles modernos. Thrinax radiata, por su parte, es explotada para construcciones rústicas, con sus troncos se fabrican trampas para langostas, y sus hojas se utilizan como techos y para hacer escobas. Ambas especies se encuentran, por tanto, seriamente amenazadas de extinción.

Algo similar ocurre con muchas especies del género Chamaedorea, principalmente aquellas que son apreciadas como ornamentales. En algunos casos es recolectada toda la planta; en otros se han sobrecolectado las semillas para exportarlas o para el cultivo, impidiendo la regeneración natural de nuevos individuos. En algunas especies se han sobrecolectado las hojas, tanto para hacer adornos florales como para exportarlas. Tal es el caso, por ejemplo, de los denominados «xiateros», que recolectan las hojas de Chamaedorea elegans, $C$. neurochlamys, $C$. seifrizii y C. oblongata. La excesiva explotación de las hojas ha provocado que muchos individuos no se desarrollen satisfactoriamente y su producción de semillas llega a ser muy escasa con la consecuente disminución de la regeneración de las poblaciones.

En México, la disminución acelerada de los bosques y selvas es un hecho evidente, que ha traído como consecuencia la pérdida de muchas especies. Esta situación, provocada por destrucción humana, ha ocasionado que grandes poblaciones de palmas ya no se recuperen porque no pueden crecer en lugares abiertos, como ha ocurrido al abrir terrenos para su cultivo, para pastizales o para crear nuevos centros urbanos. Ejemplo de esto lo tenemos en Gaussia spp., Cryosophila spp., Chamaedorea spp., Geonoma spp., Reinhardtia spp., Synechanthusfibrosus, Coccothrinax readii y Thrinax radiata.

Por los recorridos que he realizado en diferentes regiones del país, he observado que, exceptuando las plantaciones de palmas comerciales no nativas como Cocos nucifera, Phoenix dactylifera y Elaeis guineensis, no existen plantaciones de palmas mexicanas. Solamente aquellas palmas que son de utilidad para el hombre, han sido relativamente favorecidas para su explotación, como son Brahea spp., Roystonea spp., Sabal spp., Acrocomia mexicana y Scheelea liebmannii.

Es conveniente destacar que, en términos generales, no existen bancos de germoplasmas de palmas en México; sólo existen algunas colecciones en Jardines Botánicos regionales y en algunas reservas biológicas.

\section{FUTURO DE LAS PALMAS DE MÉXICO}

Debemos enfocar el futuro de las palmas de México desde dos puntos de vista: la conservación del germoplasma y su uso. En cuanto a la conservación del germoplasma, se debe 
de distinguir entre preservar y conservar. La preservación, desde mi punto de vista, debe entenderse como el mantenimiento intocable de los hábitats de las palmas, incluyéndolas a ellas mismas. Mientras que conservar se debe de entender como el mantenimiento en equilibrio del germoplasma por medio de un manejo adecuado, aun en los casos en que los recursos sean utilizados.

La preservación es una situación prácticamente imposible de realizar, porque implica mantener sin alteraciones nuestras áreas naturales, además de que cada vez existen menos regiones inalteradas en México. Aun las reservas regionales, nacionales y de la biósfera son usadas de alguna manera, ya sea de manera clandestina o por los asentamientos humanos.

Las palmas que se ven más afectadas por la alteración de los ecosistemas son las que viven en el estrato inferior y/ o medio de selvas medianas y altas, así como en bosques mesófilos, ya que la mayoría de ellas no sobreviven en condiciones de luz directa. Este es el caso de la mayoría de las especies del género Chamaedorea (el cual tiene el mayor número de especies en México, alrededor de 48), Calyptrogyne, Geonoma, Reinhardtia y Synechanthus.

Por lo anterior, se deben enfocar los esfuerzos en la conservación del germoplasma, tanto de las especies que están siendo afectadas por la alteración de los ecosistemas, como de aquellas que están siendo usadas y/o sobreexplotadas. Es interesante comentar que, a pesar de que prácticamente todas las palmas de México son usadas de alguna manera, su potencialidad de uso y de manejo es mucho mayor que el uso actual.

En México existen pocos estudios enfocados al uso y manejo de las palmas, la mayoría son muy generales y en algunos sólo se hace mención al uso de ellas. El trabajo más completo sobre un grupo de palmas es el de Caballero (1994) que hace un análisis del uso y manejo de cuatro especies de Sabal que crecen en la Península de Yucatán. Es necesario fomentar trabajos de esta naturaleza, aunados a los taxonómicos y florísticos, así como los de propagación y cultivo.

Se debe dar prioridad a aquellas palmas que se utilizan en su totalidad o en su mayor parte, como es el caso de Sabal, Brahea, Erythea, Bactris, Acrocomia, Desmoncus, Scheelea y Orbignya. Así mismo es prioritario hacer estudio de rescate, por medio del manejo del recurso, de especies que se han detectado en peligro de extinción como el caso de Coccothrinax readii, cuyos tallos están siendo cortados, en forma intensiva, para hacer construcciones rústicas en Quintana Roo, o el caso de Thrinax radiata cuyos troncos también se usan en construcción y trampas para pescar, y con cuyas hojas se hacen techos y escobas.

$\mathrm{Si}$ bien todas las palmas se caracterizan porque sus frutos y semillas tienen un alto porcentaje de aceites, las de la tribu Cocoeae son consideradas como palmeras oleíferas por su alto contenido de aceites, tanto en el mesocarpo como en el endospermo. En Sudamérica hay abundantes representantes de esta tribu, y han sido bastante estudiados, incluso hay algunos que se explotan a nivel comercial. En México también tenemos algunos, si bien menos abundantes que en Sudamérica, que forman poblaciones importantes no estudiadas aun desde el punto de vista de su contenido de aceites, y que representan por tanto un recurso de uso potencial. Entre ellos están los casos de Acrocomia, Bactris, Desmoncus, Scheelea y Orbignya.

Un aspecto desaprovechado en México es el de las palmas ornamentales, principalmente aquellas originarias de nuestro país. Exceptuando Washingtonia y Erythea, que son ampliamente cultivadas en diferentes partes del mundo, así como algunas especies de Chamaedorea, pocas son las palmas mexicanas que han sido introducidas a cultivo. Algunas especies de nuestras palmas tienen uso ornamental en las regiones cercanas a sus lugares de origen. Desafortunadamente en la mayoría de estos casos, las palmas son extraídas y trasplantadas del campo y en muy pocos casos han sido propagadas por semillas. Esto ha ocurrido con Pseudophoenix, Thrinax y Coccothrinax en la Península de Yucatán. Algo similar ha ocurrido con diversas especies de Chamaedorea en Chiapas, Oaxaca y Veracruz, causando graves daños a las poblaciones silvestres, por lo que algunas especies están actualmente en la categoría de amenazadas.

Es necesario desarrollar esfuerzos para cultivar y/o favorecer a aquellas especies en las que algunas de sus partes son recolectadas en el campo. Si bien la palma no se destruye, sí se altera considerablemente su metabolismo y en algunos casos la regeneración de las poblaciones. Ejemplos de esto lo tenemos en aquellas palmas que son usadas para techar o para elaborar diversos tipos de artesanías como Sabal y Brahea, o como los casos de Chamaedorea spp. cuyas hojas son recolectadas por los «xiateros», como ya habíamos mencionado, para su exportación y posterior uso en adornos florales, o bien para abastecer mercados nacionales. Existen poblaciones que se han hecho viejas debido a que sus frutos son recolectados intensivamente para el consumo directo, o como complemento alimenticio para el ganado como Brahea, Erythea, Roystonea, etc. En otros casos, los frutos son recolectados para su exportación, como en Chamaedorea. Esto ha provocado que las poblaciones no se renueven.

En diferentes regiones tropicales es muy frecuente la elaboración y consumo del denominado «palmito», que anatómicamente constituye la yema apical y los primordios foliares de la palma. Para elaborar este producto es necesario cortar la punta de la palma provocando su muerte. Por tanto, es necesario impedir que sean cortadas palmas silvestres como está ocurriendo en ciertas áreas de Chiapas y San Luis Potosí y, en complemento, promover grandes plantaciones para su comercialización.

El «cocotero» es una palma aparentemente originaria del Pacífico Occidental y el sudeste asiático; está ampliamente cultivado en México y en la mayoría de las regiones tropicales del mundo de manera que fisonómicamente representa fielmente el entorno tropical. Esta palmera tiene una gran cantidad de usos ya que cada una de sus partes tiene alguna utilidad. En el curso de la historia, el cocotero se ha 
visto afectado por diversas enfermedades; en épocas recientes, la enfermedad más grave que lo ha afectado es el «amarillamiento letal», que ha destruido las plantaciones de cocoteros de los llamados «altos» en el Caribe y la Florida. En México esta enfermedad llegó al principio de los 80 's, y en la actualidad ha destruido las plantaciones de la Península de Yucatán. Actualmente se encuentra en Tabasco y es probable que en algunos años destruya las poblaciones del Golfo. La enfermedad es producida por un micoplasma y no se ha encontrado ningún remedio contra ella. Las regiones donde ya no existen «cocoteros altos» se están replantando con los denominados «enanos malayos», que son cocoteros muy precoces y con una alta resistencia a la enfermedad; desafortunadamente, estas palmas no tienen las mismas características de uso que los «altos». Se siguen desarrollando los estudios para combatir al «amarillamiento letal», enfocándose hacia la genética en la búsqueda de variedades o híbridos resistentes a la enfermedad, y que presenten las características utilitarias de los «cocoteros altos».

Para finalizar y a manera de conclusión sobre el futuro de las palmas en México, debemos enfatizar la necesidad de hacer estudios interdisciplinarios entre taxónomos, etnobotánicos, ecólogos, genetistas, fisiólogos y propagadores para poder conservar estos recursos naturales reales y potenciales. Con ello, si bien es probable que los cultivos de palmas no lleguen ocupar un lugar equiparable a los principales cultígenos de México como los cereales y las legumbres, el hecho de cultivar y/o favorecer ciertas especies, podría ser el inicio para conciliar dos intereses aparentemente antagónicos en México: usar y conservar.

A continuación se enlistan algunas referencias para las personas interesadas en profundizar en algunos de los puntos mencionados en este trabajo.

Aguilar RI. 1986. Tesis de Licenciatura, Facultad de Ciencias Biológicas, Universidad Veracruzana, México. Caballero J. 1994. Doctoral Thesis. Berkeley University, California. Durán R, Franco M. 1992. Bull. Inst.fr. ètudes andinos 21:609-621. Hodel DD. 1992. Chamaedorea palms. The International Palm Society. Allen Press. Lawrence, Kansas. Olmsted I, Ercilla MJ 1988. Historia natural de las palmas chit y nakás en Quintana Roo. Serie Cuadernos de Sian Ka'an No. 2. Orellana R. 1992. Bull. Inst. fr. études andinos 21: 651-667. Orellana R, Ayora N. 1993. Principes 37: 26-34. Quero HJ. 1980. Principes 24: 118-124. Quero HJ. 1981. Principes 5: 63-72. Quero HJ. 1989. Tesis Doctoral. Facultad de Ciencias, UNAM, México. Quero HJ. 1992. Las palmas silvestres de la Península de Yucatán. Publicación especial 10, Instituto de Biología, UNAM. Quero HJ. 1992. Principes 36: 203-216. Quero HJ, Read RW. 1986. Syst. Bot 11: 145-154. Standley PC. 1920. Contr. U. S. Herb. Smit. Inst. Wash. 23: 70-84. Standley PC, Steyermark J. 1958. Fieldiana Bot. 24: 196-299. Uhl NW, Dransfield J.1987. Genera palmarum. L. H. Bailey Hortorium and The International Palm Society. Allen Press. Lawrence, Kansas. Zona S. 1990. Aliso 12: 583-666. 